Original Paper http://ajol.info/index.php/ijbcs http://indexmedicus.afro.who.int

\title{
Diversité des champignons mycorhiziens arbusculaires associés à la culture du maïs (Zea mays L.) au Bénin
}

\author{
Leslie-Dolorès Raïssa BOSSOU ${ }^{1 *}$, Howell Bidossessi HOUNGNANDAN ${ }^{1}$, \\ Appolinaire ADANDONON ${ }^{2}$, Charlotte ZOUNDJI $^{1}$ et Pascal HOUNGNANDAN ${ }^{1}$ \\ ${ }^{1}$ Laboratoire de Microbiologie des Sols et d'Ecologie Microbienne (LMSEM), Faculté des Sciences \\ Agronomiques de l'Université d'Abomey-Calavi (UAC) ; 01 BP 526 Recette Principale Cotonou, Benin. \\ ${ }^{2}$ Ecole de Gestion et de Production Végétale et Semencière, Université Nationale d'Agriculture de \\ Porto Novo ; BP : 43 Kétou, Benin. \\ *Auteur correspondant ; E-mail: bossouleslie17@gmail.com
}

\section{RESUME}

L'utilisation des champignons mycorhiziens arbusculaires (CMA) améliore durablement la baisse de la fertilité des terres et donc les rendements. Cette étude avait pour but d'évaluer la densité et la diversité des CMA associés à la culture du maïs (Zea mays L.) dans différentes zones agro-écologiques (ZAE) du Bénin. Des échantillons (sols et racines) ont été prélevés dans 38 champs répartis dans sept ZAE. Les propriétés chimiques et la densité des spores des CMA ont été déterminées à partir des échantillons de sols. La diversité des CMA a été déterminée par les indices de diversité. Les échantillons de racines ont servi à déterminer la fréquence et l'intensité de mycorhization. Les résultats obtenus ont montré la présence des CMA dans les ZAE enquêtées. Une variation de la densité en spores des CMA $(\mathrm{p}<0,05)$ par zone agro-écologique est observé avec une abondance de spore de 12501,50/100g de sol sec dans la zone cotonnière du nord Bénin (ZAE 2). Quatre genres sont identifiés au niveau de toutes les ZAE. Il s'agit des genres: Glomus, Acaulospora, Gigaspora et Scutellospora avec une dominance des Glomus (52,83\%). De plus, il existe une forte corrélation entre les paramètres chimiques et la densité de spore ainsi qu'entre les indices de diversités biologiques. Il ressort de cette étude une abondance et une diversification des CMA associés au maïs dans les différentes ZAE.

(C) 2019 International Formulae Group. All rights reserved

Mots clés : Champignons Mycorhiziens Arbusculaires, Zea mays L., diversité, zones agro-écologiques.

\section{Diversity of arbuscular mycorrhizal fungi associated with maize cropping (Zea mays L.) in Benin}

\begin{abstract}
The use of Arbuscular Mycorrhizal Fungi (AMF) could sustainably improve crop yields. This study aimed to evaluate the diversity of AMF associated with maize (Zea mays L.) production in different agroecological zones (AEZ) of Benin. Soils and root samples were collected from 38 maize fields in seven AEZ. The chemical properties and spore densities of the AMF were determined in the soil sampled. The diversity indexes were then calculated to analyze the diversity of the AMF. The collected root samples were used to determine the frequency and the intensity of mycorhization. Results from this study revealed that there was
\end{abstract}


significant difference $(\mathrm{p}<0.05)$ among agro-ecological zone (AEZ) in terms of spore density of the AMF with an abundance of spore of 12501.50/100g dry soil detected in the cotton zone of the northern Benin (AEZ 2). Four genera namely Glomus, Acaulospora, Gigaspora and Scutellospora were identified with a dominance of Glomus $(52.83 \%)$. The indexes of biological diversity (Shannon, Simpson and Hill) resulted in no significant difference ( $p>0.05$ ) among the studied AEZ. The results from the correlation test indicated a highly relationship between chemical parameters and spore density on the one hand, and between chemical parameters and indexes of biological diversity on the other hand.

(C) 2019 International Formulae Group. All rights reserved

Keywords: Arbuscular Mycorrhizal Fungus, Zea mays L., diversity, agro-ecological zones.

\section{INTRODUCTION}

Au Bénin, les activités du secteur agricole sont dominées par la production végétale marquée par une gamme de cultures vivrières (Achigan-Dako et al., 2014). Ces dernières constituent la base de la sécurité alimentaire et nutritionnelle des populations et se reposent principalement au Bénin sur la production des céréales. Selon le Ministère de l'Agriculture, de l'Elevage et de la Pêche (MAEP), la production totale de céréales est de 1814289 tonnes en 2017 contre 1643227 tonnes en 2016 soit un accroissement de $10,41 \%$. Parmi les céréales, figure le maïs (Zea mays L.) qui occupe la première place avec près de $70 \%$ des superficies céréalières emblavées (MAEP, 25010a). Il est la principale céréale intervenant dans l'alimentation au Bénin loin devant le riz et le sorgho. Outre sa fonction d'aliment de subsistance, le maïs est aussi l'objet d'échanges commerciaux tant à l'intérieur du pays que vers les marchés sous-régionaux (Boone et al., 2008).

En dépit de tous ces atouts, la filière maïs est confrontée à plusieurs problèmes qui pourraient renverser dangereusement cette tendance: il s'agit de l'inexistence d'intrants spécifiques (semences, engrais et pesticides) et la dégradation de la plupart des terres cultivables (www.procad.org). Malgré son fort potentiel de rendement, la culture du maïs est caractérisée par une faible productivité, liée à la baisse de la fertilité des sols (Lal, 2002). En effet, il est important de noter que la plupart des terres du Bénin sont dominées par les sols ferrugineux tropicaux, couvrant ainsi 60\% de la surface totale du pays (Agossou, 1983). Ces sols sont reconnus être pauvres en azote et en phosphore (Sanchez et Jama, 2002 ; Kodjo et al., 2013).

Cependant, il est important de noter l'existence de techniques pouvant permettre l'amélioration des rendements et de la qualité nutritive des sols. C'est le cas des champignons symbiotiques. Parmi ceux-ci figurent les champignons mycorhiziens à arbuscules (CMA) qui constituent le groupe le plus couramment rencontré dont les effets bénéfiques sur la croissance et la tolérance aux stress de la majorité des plantes économiquement importantes sont admis (Barea et al., 1993 ; Smith et Read, 1997 ; Banla et al., 2015). Ils permettent à la plante d'acquérir les éléments minéraux, spécialement, les éléments peu mobiles dans le sol comme le phosphore, le cuivre et le zinc (Fagbola et al., 2001; Saïdou et al., 2009; Saïdou et al., 2012 ; Haougui et al., 2013). Mais leur utilisation pratique et effective nécessite la compréhension de la diversité et de la dynamique de ces champignons dans leur environnement naturel. Ainsi, de nombreux chercheurs à travers le monde exploitent depuis très longtemps la symbiose endomycorhizienne pour améliorer la productivité de certaines plantes comme le manioc, la luzerne,...etc (Trouvelot et al., 1982 ; Aboubacar et al., 2013 ; Hamza, 2014 ; Haro et al., 2015; Do Rego et al., 2015).

Au Bénin, de nombreux travaux de recherche ont été menés par différents chercheurs sur la diversité des CMA sur différentes cultures. C'est l'exemple des travaux de recherche réalisés par Houngnandan et al. (2009) qui se sont intéressés à la diversité des espèces de glomales indigènes de la forêt claire à 
Isoberlinia doka (Craib et Stapf) à Wari-Maro au centre du Bénin. Aussi, Tchabi (2008); Johnson et al. (2013) ; Balogoun et al. (2015) ont-ils étudié la biodiversité des champignons endomycorhiziens associés respectivement à la culture du coton, du niébé et de l'anacardier. Ces différents travaux ont permis non seulement d'identifier les espèces constituant les populations de Glomeromycota associées à certaines cultures du Bénin mais aussi de montrer une diversité des espèces de ces CMA en fonction des cultures. Ces informations sont importantes pour une culture donnée, particulièrement dans une dynamique d'introduction des CMA dans une rizhosphère donnée pour la fertilité des sols. Malheureusement, de telles informations ne sont pas encore disponibles en ce qui concerne les espèces de CMA dans la rizhosphère du maïs. Aucune recherche n'a été conduite, à notre connaissance, sur les CMA associés à la culture du maïs au Bénin. Ainsi, cette étude vise à évaluer la diversité et la densité de champignons mycorhiziens arbusculaires associées à la culture du maïs, Z. mays, dans différentes zones agro écologiques du Bénin.

\section{MATERIEL ET METHODES}

\section{Zones d'étude}

Il y a au total huit zones agro écologiques au Bénin (MEHU, 2008). L'étude a été effectuée dans sept zones agroécologiques que sont : la zone extrême nordBénin (ZAE 1), la zone cotonnière du NordBénin (ZAE 2), la zone vivrière sud-Borgou (ZAE 3), la zone Ouest-Atacora (ZAE 4), la zone cotonnière du Centre-Bénin (ZAE 5), la zone des terres de barre (ZAE 6) et la zone des pêcheries (ZAE 8). La zone des terres de barres (ZAE 7) n'a pas été prospectée.

\section{Prélèvement de sol}

Ces prélèvements ont été effectués durant le mois d'Août 2017 dans les différentes zones agro-écologiques sus mentionnées. Au moins deux localités ont été choisies par ZAE. Ces localités ont été choisies en tenant compte de leur type de sol afin d'avoir une variabilité d'échantillons. Dans chaque localité, deux différents champs ont été prospectés et au niveau de chaque champ quatre échantillons ont été prélevés à raison d'un échantillon par plant de maïs. Un cadran d'un mètre carré a été posé au niveau du plant de maïs choisi et l'échantillon est prélevé dans ce cadran. Le prélèvement d'échantillon de sol se fait à l'aide d'une sonde à $20 \mathrm{~cm}$ de profondeur. Les quatre échantillons de sol prélevés dans le champ sont mis ensemble pour constituer un échantillon composite de $500 \mathrm{~g}$. Cet échantillon composite de $500 \mathrm{~g}$ de sols ainsi constitué par site a été utilisé pour l'extraction de spores en vue de la détermination de la densité et de la diversité sporale des champignons mycorhiziens à arbuscules ainsi que pour les analyses chimiques.

\section{Prélèvement des échantillons de racines}

Quatre plants de maïs situés dans l'environnement le plus immédiat des points de prélèvements des sols (04) ont été soigneusement déterrés. Leurs racines ont été coupées à l'aide d'un couteau et mises dans un sachet plastic étiqueté.

\section{Analyse microbiologique des sols Extraction des spores}

L'extraction des spores a été faite suivant la méthode de tamisage humide décrite par Gerdemann et Nicholson (1963). Un échantillon de $100 \mathrm{~g}$ de sol sec a été mis en suspension dans $500 \mathrm{ml}$ d'eau de robinet. La suspension obtenue a été transvasée dans une série de quatre tamis à mailles décroissantes $(250 \mu \mathrm{m}, 150 \mu \mathrm{m}, 63 \mu \mathrm{m}$ et 50 $\mu \mathrm{m})$. Il a été ajouté au filtrat, obtenu par tamis, $5 \mathrm{ml}$ de deux solutions chacune de concentrations différentes de saccharose $(20 \%$ et $60 \%$ ), et le mélange est mis dans une centrifugeuse pendant 3 min à 4000 tours par minute et à une température de $4{ }^{\circ} \mathrm{C}(\mathrm{Oehl}$ et al., 2003).

\section{Dénombrement des spores}

L'abondance des spores a été évaluée sous une loupe binoculaire à partir du filtrat à saccharose centrifugé et mis dans une boîte de Pétri $(5 \mathrm{~cm}$ de diamètre) quadrillée. Les spores ont été comptées en fonction de leurs tailles, de leurs couleurs puis du mode d'attachement de l'hyphe à la spore. L'identification et la description des espèces répertoriées ont été faites en utilisant la clé 
d'identification de l'«International Culture Collection of Vesicular and arbuscular Mycorrhizal fungi» (INVAM, http//www.invam.caf.wdu.edu). Différents indices de diversité biologiques ont été calculés sur la base des nombres de spores obtenues par type de couleur et de différenciation. Il s'est agi de l'indice de diversité de Shannon (H') (Shannon et Weaver, 1962), de l'indice de diversité de Simpson (1-D) (Simpson, 1949) et de l'indice de diversité de Hill (1-Hill). Ces indices ont été utilisés afin d'extraire le maximum d'informations et de mieux comprendre les communautés de CMA.

\section{Evaluation des paramètres de mycorhization}

Les racines fines $\mathrm{du}$ maïs préalablement mises dans une solution de $\mathrm{KOH} 10 \%$, ont été colorées dans du Bleu trypan $(0,05 \%)$. Ces racines colorées ont été découpées en fragments d'un centimètre et ont été montées entre lames et lamelles. La fréquence et l'intensité de mycorhization ont été évaluées au microscope optique en utilisant la méthode de Trouvelot et al. (1986).

\section{Analyse chimique des sols}

Les analyses chimiques de sol ont porté sur la teneur en carbone organique fait par la méthode de Walkley et Black (1934), le phosphore assimilable par la méthode de Bray et Kurtz (1945) et le $\mathrm{pH}_{\mathrm{H} 2 \mathrm{O}}$ par la méthode potentiométrique (Mallouhi, 1997).

\section{Analyse statistique}

Les données issues de l'évaluation de la densité et de l'étude de la diversité de spore ont été soumises à des analyses de variances à un facteur (ZAE) grâce au logiciel SAS version 9.2. Ces analyses ont permis de faire ressortir l'existence ou non de différence significative entre les ZAE. Le test de Student Newman-Keuls au seuil de 5\% a été utilisé pour la séparation des moyennes. Une analyse factorielle en composante (AFC) a été effectuée grâce aux données issues de la diversité de spore. Elle a permis d'obtenir la caractérisation des genres de CMA par ZAE. Les données issues des paramètres chimiques ont été utilisées pour voir la relation existante entre eux et la densité d'une part et la diversité d'autre part.

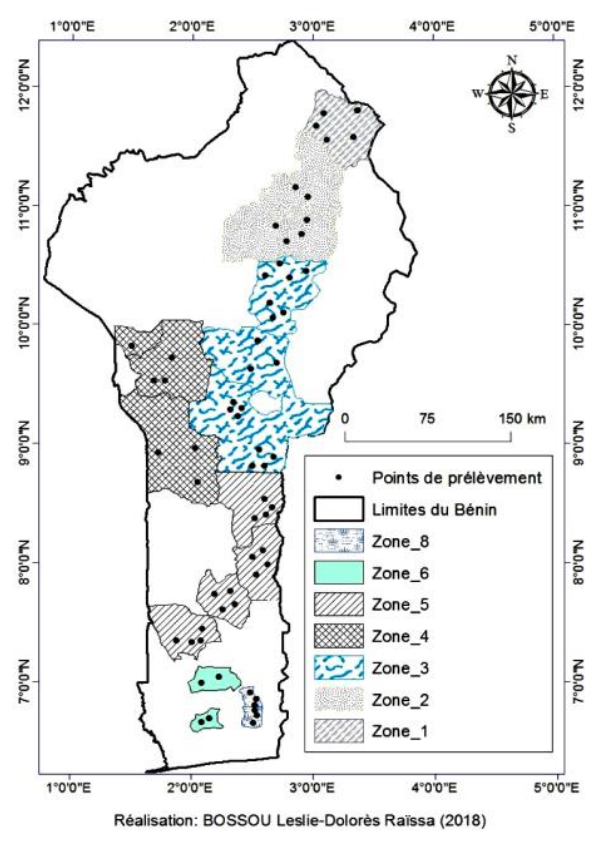

Figure 1 : Présentation des zones de l'étude. 


\section{RESULTATS}

\section{Densité des CMA associés au maïs dans} différentes zones agro-écologiques du Bénin

L'extraction de spore des différents sols a permis de connaitre la densité en spore des sols par zone agro-écologique consignées dans le Tableau 1. Il en ressort une différence très hautement significative $(p<0,001)$ entre les différentes ZAE. En effet, la comparaison des moyennes entre les différentes ZAE a montré que la ZAE 2 comporte un grand nombre de spores (1252 par $100 \mathrm{~g}$ de sol sec) et est donc la plus dense.

Les spores de CMA observées au niveau de différents sols associés à la culture du maïs étaient de couleurs variées à savoir : noire, brune, blanche, et jaune. Une analyse comparative des moyennes de densité de spore en fonction des couleurs par ZAE (Tableau 2) montre une différence très hautement significative $(p<0,001)$ entre les ZAE du point de vue couleur de spores. De façon générale, la densité des spores de couleur noire est la plus importante dans toutes les ZAE, puis viennent ensuite celles de couleur blanche. La ZAE ayant une bonne densité de spores au niveau de toutes les couleurs est la ZAE 2. Le Tableau 3 quant à lui, présente le nombre moyen de spores par $100 \mathrm{~g}$ de sol sec selon leur taille déterminée en utilisant des tamis dont les diamètres de mailles ont été de $250 \mu \mathrm{m}, 150 \mu \mathrm{m}, 63 \mu \mathrm{m}$ et $50 \mu \mathrm{m}$. Il existe de différence significative au seuil de 5\% entre les ZAE du point de vue nombre de spores par taille. On en déduit que la densité des spores est plus abondante pour les CMAs de petite taille quelques soient les ZAE.

\section{Diversité des CMA associés au maïs dans différentes ZAE du Bénin}

L'évaluation de la diversité prend en compte ici les indices de diversité et l'abondance relative. Il n'existe pas de différence significative (au seuil de 5\%) entre les ZAE concernant tous les indices
(Shannon-Weiner, Simpson et Hill) (Tableau 4). La valeur inférieure de l'indice de diversité de Shannon est de 1,991 (ZAE 8) tandis que la valeur supérieure est de 2,359 (ZAE 1). Ces valeurs indiquent que la communauté de CMA est dans une certaine mesure diversifiée en fonction des ZAE même si la différence n'est pas statistiquement significative. Par contre, l'indice de Hill (1-H) varie entre 0,8246 (ZAE 4) et 0,89476 bit (ZAE 1). Ces faibles valeurs de cet indice indiquent que la communauté de CMA est très peu diversifiée suivant les zones agro-écologiques. L'indice de Simpson (1-D) varie de 0,80579 à 0,898; ce qui implique que les CMA sont diversifiée d'une ZAE à une autre. Après analyse de ces différents échantillons de sol, il a été globalement remarqué une dominance de 4 (quatre) genre d'espèces que sont : Glomus en moyenne 52,82\%, par rapport à celles des Gigaspora (23,59\%), des Scutellospora (14,99\%) et celles des Acaulospora (8,60\%).

\section{Relation existante entre les paramètres chimiques, la densité et la diversité des spores associés à la culture du maïs}

De façon globale, il existe une forte relation entre les paramètres chimiques et la densité totale des spores dans les ZAE ainsi qu'entre les paramètres chimiques et l'indice de diversité de Shannon dans toutes les ZAE.

\section{Typologie des ZAE basée sur les espèces de CMA}

Cette typologie vise à regrouper les ZAE par groupe d'espèces de CMA. Les deux premières composantes, expriment $66,97 \%$ $(>50 \%)$ de l'information du départ. Ce qui est suffisant pour garantir la fiabilité de l'interprétation des résultats.

Les résultats de l'AFC (Figure 2) montrent que les ZAE 1,3 , 4 et 5 sont corrélées de façon positive par la dominance des espèces du genre Glomus et de Gigaspora. Les espèces du genre Acaulospora, Scutelospora et Glomus sont très bien 
représentées négativement sur l'axe1 et caractérisent donc, la ZAE 2. La ZAE 7 est caractérisée par les espèces du genre Glomus et du genre Scutellospora. La ZAE 6 est caractérisée quant à elle par les espèces du genre Gigaspora et Acaulospora qui est représenté positivement sur l'axe 1 .
Mycorhization naturelle des racines de maïs

Les résultats révèlent qu'il $\mathrm{y}$ a une différence significative entre les ZAE ( $\mathrm{p}<$ $0,001)$ avec une forte fréquence de mycorhization de la ZAE $4(20,42 \%)$ par rapport aux autres. De plus, la moyenne des intensités de mycorhization des racines de maïs de la ZAE 4 sont plus intensément mycorhizées $(2,47)$ que les autres au seuil de 5 $\%$.

Tableau 1 : Densité moyenne en spores par 100g de sols au niveau de chaque zone agroécologique.

\begin{tabular}{lll}
\hline Facteurs d'étude & Modalités & Densité moyenne de spore \\
\hline \multirow{2}{*}{ ZAE } & ZAE 1 & $4994,50 \pm 46,50 \mathbf{b}$ \\
\cline { 2 - 3 } & ZAE 2 & $12501,50 \pm 1850,50 \mathbf{a}$ \\
\cline { 2 - 3 } & ZAE 3 & $7924,75 \pm 418,05 \mathbf{a b}$ \\
\cline { 2 - 3 } & ZAE 4 & $5826,67 \pm 234,34 \mathbf{b}$ \\
\hline ZAE 5 & $8919,75 \pm 2090,54 \mathbf{a b}$ \\
\cline { 2 - 3 } & ZAE 6 & $4894,00 \pm 82,00 \mathbf{b}$ \\
\cline { 2 - 3 } & ZAE 8 & $3259,00 \pm 32,00 \mathbf{b}$ \\
\hline
\end{tabular}

Les nombres moyens suivis des mêmes lettres alphabétiques ne sont pas significativement différentes $(P>0,05)$ d'après le test de Student Newman-Keuls.

Tableau 2 : Densités moyennes en spores des sols suivant la couleur sous culture de maïs dans les différents ZAE.

\begin{tabular}{clcccc}
$\begin{array}{l}\text { Facteurs } \\
\text { d'étude }\end{array}$ & Modalités & brun & blanc & noir & jaune \\
\hline \multirow{2}{*}{ ZAE } & ZAE 1 & $1175,50 \pm 80,50 \mathbf{a b}$ & $1394,50 \pm 181,50 \mathbf{b c}$ & $1803,50 \pm 263,50 \mathbf{e}$ & $721,00 \pm 118,00 \mathbf{a b}$ \\
\cline { 2 - 6 } & ZAE 2 & $1963,50 \pm 103,50 \mathbf{a}$ & $3856,00 \pm 68,00 \mathbf{a}$ & $4982,50 \pm 41,50 \mathbf{a}$ & $799,50 \pm 37,50 \mathbf{a}$ \\
\cline { 2 - 6 } & ZAE 3 & $1361,50 \pm 126,31 \mathbf{a b}$ & $2440,25 \pm 162,34 \mathbf{b}$ & $3390,75 \pm 104,07 \mathbf{c}$ & $632,00 \pm 70,29 \mathbf{a b}$ \\
\cline { 2 - 6 } & ZAE 4 & $906,00 \pm 94,85 \mathbf{a b}$ & $1739,33 \pm 59,49 \mathbf{b c}$ & $3946,50 \pm 12,50 \mathbf{b c}$ & $129,00 \pm 14,00 \mathbf{d}$ \\
\cline { 2 - 6 } & ZAE 5 & $1853,50 \pm 362,36 \mathbf{a}$ & $2311,75 \pm 487,50 \mathbf{b}$ & $4332,25 \pm 231,53 \mathbf{a b}$ & $489,25 \pm 45,81 \mathbf{b c}$ \\
\cline { 2 - 6 } & ZAE 6 & $1141,00 \pm 114,00 \mathbf{a b}$ & $717,50 \pm 11,50 \mathbf{c}$ & $2644,50 \pm 322,50 \mathbf{d}$ & $334,00 \pm 5,00 \mathbf{c}$ \\
\cline { 2 - 6 } & ZAE 8 & $331,00 \pm 28,50 \mathbf{b}$ & $700,00 \pm 38,00 \mathbf{c}$ & $2152,00 \pm 97,00 \mathbf{d e}$ & $75,50 \pm 2,50 \mathbf{d}$ \\
\hline
\end{tabular}

Les nombres moyens suivis des mêmes lettres alphabétiques ne sont pas significativement différentes $(P>0,05)$ d'après le test de Student Newman-Keuls. 
Tableau 3: Densités moyennes en spores des sols suivant leur taille sous culture du maïs dans les différentes ZAE.

\begin{tabular}{cccccc}
\hline $\begin{array}{c}\text { Facteurs } \\
\text { d'étude }\end{array}$ & Modalités & $\mathbf{2 5 0} \boldsymbol{\mu m}$ & $\mathbf{1 5 0} \boldsymbol{\mu m}$ & $\mathbf{6 3 \mu \mathbf { m }}$ & $\mathbf{5 0 \mu \mathbf { m }}$ \\
\hline & ZAE 1 & $765.50 \pm 8,50 \mathbf{a}$ & $1351,00 \pm 14,00 \mathbf{c}$ & $1085,50 \pm 51,50 \mathbf{b}$ & $2242,50 \pm 77,50 \mathbf{a}$ \\
\cline { 2 - 6 } & ZAE 2 & $738,00 \pm 25,00 \mathbf{a}$ & $3235,50 \pm 258,50 \mathbf{a}$ & $4696,50 \pm 409,50 \mathbf{a}$ & $2430,50 \pm 256,50 \mathbf{a}$ \\
\cline { 2 - 6 } & ZAE 3 & $900,50 \pm 106,78 \mathbf{a}$ & $2489,33 \pm 63,29 \mathbf{b}$ & $3786,25 \pm 202,01 \mathbf{a}$ & $1089,25 \pm 39,21 \mathbf{b}$ \\
\cline { 2 - 6 } ZAE & ZAE 4 & $339,33 \pm 12,41 \mathbf{b c}$ & $2149,00 \pm 15,72 \mathbf{b}$ & $2043,00 \pm 11,14 \mathbf{a b}$ & $1262,00 \pm 74,87 \mathbf{b}$ \\
& ZAE 5 & $591,33 \pm 35,36 \mathbf{a b}$ & $3071,00 \pm 258,89 \mathbf{a}$ & $4236,75 \pm 970,01 \mathbf{a}$ & $\begin{array}{c}1541,00 \pm \\
285,74 \mathbf{b}\end{array}$ \\
\cline { 2 - 6 } & ZAE 6 & $376,50 \pm 6,50 \mathbf{b c}$ & $969,50 \pm 116,50 \mathbf{c}$ & $\begin{array}{c}2664,00 \pm \\
202,00 \mathbf{a b}\end{array}$ & $884,00 \pm 3,00 \mathbf{b}$ \\
\cline { 2 - 6 } & ZAE 8 & $212,00 \pm 22,00 \mathbf{c}$ & $1318,50 \pm 133,50 \mathbf{c}$ & $706,00 \pm 96,00 \mathbf{b}$ & $1023,00 \pm 10,00 \mathbf{b}$ \\
\hline
\end{tabular}

Les nombres moyens suivis des mêmes lettres alphabétiques ne sont pas significativement différentes $(P>0,05)$ d'après le test de Student Newman-Keuls.

Tableau 4 : Valeurs des indices de diversité des zones agro-écologiques (ZAE).

\begin{tabular}{ccccc}
\hline $\begin{array}{c}\text { Facteurs } \\
\text { d'étude }\end{array}$ & Modalités & H' & 1-D & 1-H \\
\hline \multirow{2}{*}{ ZAE } & ZAE 1 & $2,359 \pm 0,034 \mathrm{a}$ & $0,89844 \pm 0,0061 \mathrm{a}$ & $0,89476 \pm 0,004316 \mathrm{a}$ \\
\cline { 2 - 5 } & ZAE 2 & $2,124 \pm 0,29 \mathrm{a}$ & $0,85912 \pm 0,0476 \mathrm{a}$ & $0,85663 \pm 0,0485 \mathrm{a}$ \\
\cline { 2 - 5 } & ZAE 3 & $2,061 \pm 0,281 \mathrm{a}$ & $0,88759 \pm 0,05298 \mathrm{a}$ & $0,85301 \pm 0,03678 \mathrm{a}$ \\
\cline { 2 - 5 } & ZAE 4 & $1,967 \pm 0,238 \mathrm{a}$ & $0,82528 \pm 0,0557 \mathrm{a}$ & $0,82466 \pm 0,05604 \mathrm{a}$ \\
\cline { 2 - 5 } & ZAE 5 & $2,149 \pm 0,107 \mathrm{a}$ & $0,85028 \pm 0,02791 \mathrm{a}$ & $0,86183 \pm 0,01936 \mathrm{a}$ \\
\cline { 2 - 5 } & ZAE 6 & $2,105 \pm 0,215 \mathrm{a}$ & $0,83891 \pm 0,0528 \mathrm{a}$ & $0,85174 \pm 0,0406 \mathrm{a}$ \\
\hline
\end{tabular}

Tableau 5 : Valeurs des coefficients de corrélation par ZAE.

\begin{tabular}{cccccccc}
\hline $\begin{array}{c}\text { Coefficient de } \\
\text { Pearson R }\end{array}$ & ZAE 1 & ZAE 2 & ZAE 3 & ZAE 4 & ZAE 5 & ZAE 6 & ZAE8 \\
\hline P x densité totale & ns & $0,965^{*}$ & 0,980 & $0,950^{*}$ & $0,986^{*}$ & $0,954^{*}$ & $0,988^{*}$ \\
\hline MO x densité totale & $0,959^{*}$ & $0,987^{*}$ & ns & $0,962^{*}$ & $0,954^{*}$ & $0,972^{*}$ & ns \\
\hline $\mathbf{p H}_{\mathbf{H 2 O}} \mathbf{x}$ densité totale & $0,956^{*}$ & $0,997^{* *}$ & $0,987^{*}$ & $\mathrm{~ns}$ & $-0,974^{*}$ & $-0,983^{*}$ & $\mathrm{~ns}$ \\
\hline
\end{tabular}




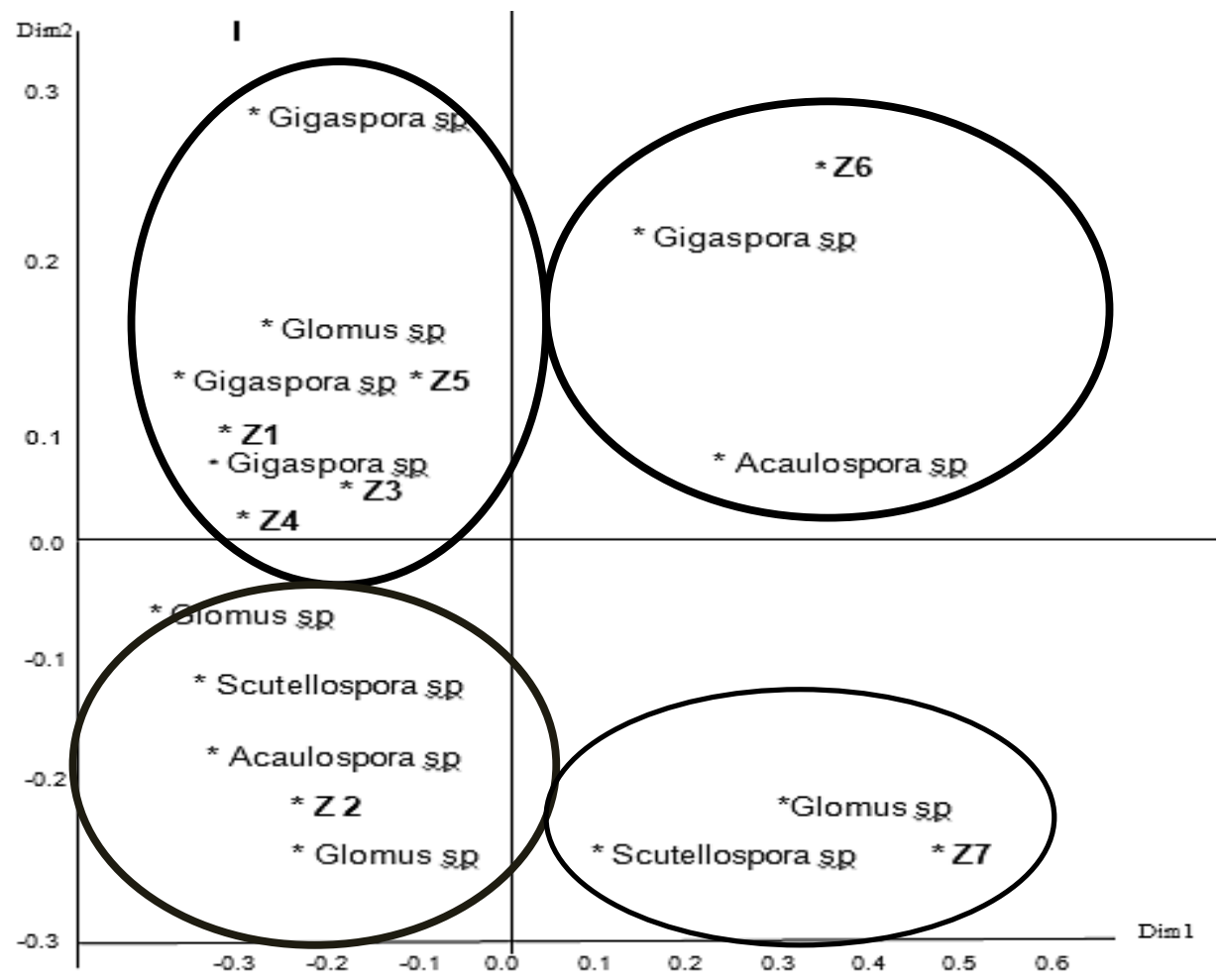

Figure 2 : Caractérisation des espèces de CMA par ZAE.

\section{DISCUSSION}

\section{Densité en spores de CMA associée à la culture du maïs}

Les résultats issus de ces travaux, révèlent une densité en spores très élevée (environ 134156 spores pour $100 \mathrm{~g}$ de sol) pour toutes les ZAE. Cette densité est nettement supérieure à celle obtenue par Johnson et al. (2013) (environ 4045 spores pour $100 \mathrm{~g}$ de sol) sous culture de niébé dans les différentes zones agro- écologiques du Bénin, par Bivoko et al. (2013) sous culture de manioc dans la région d'Azaguié au SudEst de la Cote d'Ivoire, par Balogoun et al. (2015) sous plantation d'anacarde au centre du Bénin ainsi qu'à celle retrouvée dans la forêt classée de Wari-Maro au Nord-Bénin sous Isoberlinia doka par Houngnandan et al. (2009). Les racines du maïs sont moins abondantes, trapues et dépourvues de poils absorbants donc particulièrement dépendantes des CMA (Garbaye, 2013). Le système racinaire de la culture du maïs est caractérisé par la présence de racines adventives qui permettent de puiser leurs éléments nutritifs uniquement dans les horizons superficiels. Ainsi, les CMA permettent le développement des racines afin qu'elles puissent améliorer le prélèvement d'eau et de nutriments dans le sol. Contrairement aux travaux de Johnson et al. (2013) faisant remarquer l'inexistence de différence significative entre les différentes ZAE du Bénin concernant la densité en spores de Glomeromycota associés au niébé, les résultats issus de la présente étude montrent que la ZAE 2 c'est-à-dire la zone cotonnière du nord Bénin est significativement différente des autres ZAE suivies par les ZAE 3 et 5 . Il est à noter que ces ZAE sont des zones de culture du coton et donc en perpétuel utilisation, ce qui pourrait favoriser le développement des microorganismes. De plus, cette différence pourrait être aussi due à la période de prélèvement des échantillons de sol et des précédents culturaux qui sont des paramètres important à considérer dans 
l'évaluation de la densité des spores dans le sol. Selon Jacobson (1997) et Bohrer et al. (2003), le nombre de spores est plus élevé dans le sol après que ce dernier soit soumis à des conditions de stress hydrique d'une durée relativement longue (saison sèche) et ce, grâce à la production de spores (structures de résistance). Il en découle que le climat à travers les saisons serait un des facteurs à la base de l'abondance de spores de mycorhizes arbusculaires. Les spores de petites et moyennes tailles sont les plus abondantes que celles de grandes tailles dans toutes les ZAE étudiées. Ces résultats sont conformes à ceux de Johnson et al. (2013) et ceux de Dione (2007) qui remarquaient que l'effectif des spores de CMA est inversement proportionnel à leur taille.

\section{Diversité en spores de CMA associée à la culture du maïs}

Douze espèces réparties en quatre genres : Glomus, Acaulospora, Gigaspora et Scutellospora ont été identifiées sous culture du maïs dans l'ensemble des ZAE. Cette richesse spécifique est supérieure à celle obtenue pour d'autres cultures au Bénin par Balogoun et al. (2015) ainsi que par Houngnandan et al. (2009). Cette richesse spécifique est par contre, inférieure à celle obtenue sous culture de niébé (15 espèces) par Johnson et al. (2013) dans l'ensemble des ZAE du Bénin et celle rencontrée par Diop et al. (2014) au Sénégal (15 espèces). La richesse spécifique varierait donc d'une culture à une autre. Les quatre genres de spores de CMA retrouvés durant cette étude sont identiques à ceux identifiés par Mbogne et al. (2015) et Johnson et al. (2013). Ces quatre genres sont généralement ceux identifiés, dans la rhizosphère d'autres cultures, par différents microbiologistes des régions ouest-africaines et centrales (Ngonkeu et al., 2003) et par d'autres de la région méditerranéenne (Bouamri et al., 2006) en zone forestière humide au Cameroun et en zone soudanienne au Burkina-Faso. Les résultats rapportés dans la présente étude indiquent que la diversité en spores de champignons varie peu d'un agroécosystème à un autre avec une dominance des spores du genre Glomus extraites des sols des différentes ZAE. La prédominance des espèces du genre Glomus dans la plupart des écosystèmes suggère une meilleure adaptation de ce genre soit aux conditions les plus hostiles telles que la sécheresse, la salinité et autres stress environnementaux (Blaszkowski et al., 2002), ou à une large gamme de niches écologiques (Houngnandan et al., 2009). En effet, les genres Glomus se propageraient beaucoup plus par les spores qui sont des formes de résistance des CMA aux conditions difficiles tandis que les genres Gigaspora et Scutellospora se propageraient davantage avec d'autres types de propagules tels que les hyphes et les fragments mycéliens extraracinaires (Brito et al., 2012). Les résultats concernant l'indice de diversité indiquent que la communauté de CMA est très diversifiée quelque soient les ZAE.

\section{Colonisation racinaire du maïs}

Les fréquences de mycorhization des racines de maïs obtenues au cours de cette étude sont d'environ $20 \%$ et présentent de différence significative d'une zone agroécologique à une autre. Les intensités de mycorhization sont très faibles et inférieures à $2,47 \%$ dans les ZAE. Ces résultats sont contraires à ceux obtenus par Houngnandan et al. (2009) qui ont observé un taux de mycorhization variant de $15,46 \%$ à $63,15 \%$, et par Johnson et al. (2013) qui observe une variation de la fréquence de mycorhization $45 \%$ à la floraison à $60 \%$ à la fructification du niébé. Cela pourrait être dû au fait que l'infection mycorhizienne des plantes varie significativement d'une plante à une autre, au sein de la même espèce, mais aussi selon les génotypes de l'espèce, les conditions environnementales, l'âge de la plante, la teneur en azote etc. (Duponnois et al., 2001).

\section{Impact des paramètres chimiques sur la densité et la diversité des CMA dans les ZAE étudiés}

Les résultats issus de cette étude montrent l'existence d'une forte relation entre les paramètres chimiques et la densité totale 
des spores dans les ZAE. Ces résultats sont conformes à ceux de Saïdou (2009). Il existe majoritairement une corrélation positive entre le $\mathrm{P}$ assimilable, la matière organique et la densité des spores. En effet, le rôle majeur des mycorhizes se situe au niveau de la mobilisation pour la plante d'éléments nutritifs très peu mobiles dans le sol, principalement le phosphore (Duponnois et al., 2000; Lambers et al., 2008). En fonction du $\mathrm{pH}$ du sol, cet élément se retrouve majoritairement piégé par le fer, l'aluminium ou le calcium sous des formes difficilement mobilisables par les plantes (Hinsinger, 2001). Il a également été démontré que les associations mycorhiziennes pouvaient jouer un rôle significatif dans la décomposition et la minéralisation des matières organiques végétales et mobiliser les nutriments au bénéfice de la plante hôte (Gobat et al., 2003 ; Larnbers et al., 2008). Selon Amijee et al. (1989), le phosphore peut être un facteur limitant de l'abondance de spores, que ce soit à trop forte concentration ou trop faible concentrations (Lagrange, 2009). Par conséquent il est nécessaire de déterminer la bonne concentration en cet élément avant tout apport d'inoculum mycorhiziens.

\section{Conclusion}

L'objectif global de cette étude a été de mettre en évidence la diversité des champignons mycorhiziens arbusculaires (CMA) associés à la culture du maïs dans différentes zones agro- écologiques du Bénin. A travers les résultats obtenus, il apparaît que la densité en spores des CMA diffère d'une ZAE à une autre avec une prédominance au niveau de la zone cotonnière du nord Bénin (ZAE 2). La fréquence et l'intensité de mycorhization des racines de maïs sont relativement très faibles dans l'ensemble des ZAE ; ce qui dénote du faible pouvoir d'infection des CMA indigènes. De plus, les spores de couleur noire sont les plus abondantes suivies de celles blanches. On observe également une abondance de spores au niveau des mailles de petites et moyennes tailles $(50 \mu \mathrm{m}, 63 \mu \mathrm{m}$ et $150 \mu \mathrm{m})$. La diversité en spores de champignons varie peu d'une
ZAE à une autre. Ces résultats ont identifié 12 espèces réparties en quatre genres : Glomus, Acaulospora, Gigaspora et Scutellospora dans l'ensemble des ZAE avec une abondance du genre Glomus. Il existe une forte relation entre les paramètres chimiques et la densité totale des spores dans les ZAE ainsi qu'avec l'indice de diversité de Shannon. Il ressort de cette étude une abondance et une diversification des CMA associés au maïs dans les différentes ZAE. Cela permettra la production d'inoculum mycorhiziens en vue d'accroitre la productivité du maïs.

\section{CONFLITS D'INTERETS}

Les auteurs de cet article déclarent qu'il n'existe aucun conflit d'intérêts.

\section{CONTRIBUTIONS DES AUTEURS}

Tous les auteurs ont participé activement à la réalisation de cette étude et à la rédaction du manuscrit.

\section{REMERCIEMENTS}

Ce travail a été entièrement financé par le Laboratoire de Microbiologie des Sols et d'Ecologie Microbienne (LMSEM) de la Faculté des Sciences Agronomiques (FSA). Un sincère remerciement à AKPLO Moriaque, pour son aide dans la réalisation des analyses statistiques.

\section{RÉFÉRENCES}

Aboubacar K, Ousmane ZM, Amadou HI, Issaka S, Zoubeirou AM. 2013. Effet de la co-inoculation du rhizobium et de mycorhizes sur les performances agronomiques du niébé au Niger. Journal of Applied Biosciences, 72(1): 5846- 5854.

Achigan-Dako EG, Houdegbe AC, Glèlè M, Womdim RN. 2014 Analyse du système de production et de distribution des semences de maïs Zea mays L. au SudBénin. Biotechnoogiel. Agronomie. Société Environnement., 18(1) : 49-60.

Balogoun I, Saïdou A, Kindohoundé NS, Ahoton EL, Amadji GL, Ahohuendo BC, 
Babatoundé S, Chougourou D, BabaMoussa L, Ahanchédé A. 2015 Soil Fertility and Biodiversity of Arbuscular Mycorrhizal Fungi Associated with Cashew's Anacardium occidentale L. Cultivars Characteristics in Benin West Africa. International Journal of Plant and Soil Science, 51: 50-63.

Banla EM, Agnassim BANITO A, Sogbedji JM. 2015. Effects of Arbuscular Mycorrhizal Fungi on the production of tomato in Togo. International Journal of Biological and Chemical Sciences, 9(3): 1270-1276.

DOI: http://dx.doi.org/10.4314/ijbcs.v9i3.12

Barea JM, Honrubia M. 1993. Micorrizas y revegetaciôn. Ecosistemas, 4: 46-47.

Bivoko DR, Ahonzo-Niamke SL, Zeze A. 2013. Impact des propriétés physicochimiques des sols de culture du manioc sur l'abondance et la diversité des communautés de champignons mycorhiziens à arbuscules dans la zone agroécologique d'Azaguie, sud-est de la Côte d'Ivoire. Agronomie Africaine, 25(3): 251-264.

Bohrer G, Kangan-Zur V, Roth-Bejerano N, Ward D, Beck G, Bonifacio E. 2003. Effect of different Kalahari-desert VA mycorrhizal communities on mineral acquisition and depletion from soil by hast plants. Journal of Arid Environment, 55: 193-208. DOI: 10.1016/S01401963(03)00047-8

Boone P, Stathacos CJD, Wanzie RL. 2008. Évaluation sous-régionale de la chaine de valeurs du maïs. Rapport technique ATP $n^{\circ}$ 1. Bethesda, MD, USA:AbtAssociates Inc.

Bouamri R, Dalpé Y, Serrhini MN, Bennani A. 2006 Arbuscular mycorrhizal fungi species associated with rhizosphere of Phoenix dactylifera L. in Morocco. African Journal of Biotechnology, 5(6): 510-516.
Bray RH, Kurtz LT. 1945 Determination of total, organic, and available forms of phosphorus in soils. Soil Science, 59(1): $39-45$

Dione B. 2007. Valorisation des communautés de champignons mycorhiziens associés aux plantes pionnières dans la domestication des essences forestières en milieu sahélien. Diplôme national de master en sciences et technologie. 46p.

Diop TA, Gueye M, Dreyfus BL, Plenchette C, Strullu DG. 1994. Indigenous arbuscular mycorrhizal fungi associated with Acacia albida in different areas of Senegal. Applied and Environmental Microbiology, 60: 3433-3436.

Do Rego FA, Diop I, Sadio O, Da Sylva MC, Agbangba CE, Touré O, Kane A, Neyra M, Ndoye I, Krasova-Wade T. 2015. Response of cowpea to symbiotic microorganisms inoculation Arbuscular mycorrhizal fungi and Rhizobium in cultivated soils in Senegal. Universal Journal of Plant Science, 32: 32-42. DOI: 10.13189/ujps.2015.030204.

Duponnois R, Plenchette C, Thioulouse J, Cadet P. 2001. The mycorrhizal soil infectivity and arbuscular mycorrhizal fungal spore communities in soils of different aged fallows in Senegal. Applied Soil Ecology, 17: 239-251. DOI: S0929-1393(01)00132-9.

Fagbola O, Osonubi O, Mulongoy K, Odunfa SA. 2001. Effects of drought stress and arbuscular mycorrhiza on the growth of Glirincidiasepium Jacq,Walp and Leucaena leucocephala Lam de Wit, in simulated eroded soil conditions. Mycorrhiza, 11(5): 215-223. DOI: http ://doi.org/10.1007/s005720100114.

Garbaye J. 2013. La symbiose mycorhizienne, Quae, $280 \mathrm{p}$.

Gerdemann JW, Nicholson TH. 1963. Spores of mycorrhizal Endogone species extracted from soil by wet sieving and 
decanting. Transactions of British Mycological Society, 46: 235-244.

Haro H, Sanon KB, Krasova-Wade T, Kane A, N'doye I, Traore AS. 2015. Réponse à la double inoculation mycorhizienne et rhizobienne du niébé variété, KVX3964- 5-2D cultivé au Burkina Faso. International Journal of Biological and Chemical Sciences, 9(3): 1485-1493. DOI:

http://dx.doi.org/10.4314/ijbcs.v9i3.31.

Houngnandan P, Yemadje RGH, Kane A, Boeckx P, Van Cleemput O. 2009. Les glomales indigènes de la forêt claire à Isoberlinia dokaCraibet Stapf à WariMaro au centre du Bénin. Tropicultura, 27(2): 83-87.

Jacobson KM. 1997. Moisture and substrate stability determine VA-mycorrhizal fungal community distribution and structure in arid grassland. Journal of Arid Environments, 35:59-75.

Johnson JM, Houngnandan P, Kane A, Sanon K, Neyra M. 2013. Diversity patterns of indigenous arbuscular mycorrhizal fungi associated with rhizosphere of cowpea Vigna unguiculata L. Walp. in Benin, West Africa. Pedobiologia, 56(3): 121128.

DOI:

https://doi.org/10.1016/j.pedobi.2013.03. 003.

Kodjo S, Adjanohoun A, Akondé TP, Aïhou K, Kpagbin G, Gotoechan H, Igue AM. 2013. Diagnostic participatif de la fertilité des sols des exploitations agricoles à base de maïs Zea mays dans les départements du Zou et des Collines au Bénin. Bulletin de la Recherche Agronomique du Bénin BRAB, 2013 : 3953.

Kombienou PD, Arouna O, Azontondé AH, Mensah GA, Sinsin BA. 2015. Caractérisation du niveau de fertilité des sols de la chaîne de l'Atakora au nordouest du Bénin. Journal of Animal and Plant Sciences, 25: 3836-3856.
Lambert DH, Baker DE, Cole JH. 1979. The role of mycorrhizae in the interactions of phosphorus with zinc, copper, and other Elements. Soil Science Society of America, 43: 976-680.

Lambert DH, Weidensaul TC, Borgerand DC, Rhode LH. 1985. Use of sewage sludge for forest tree seedling production. United States Environmental Protection Agency, 6: 32-85.

Mbogne TJ, Temegne C, Hougnandan P, Youmbi E, Tonfack LB, NtsombohNtsefong G. 2015. Biodiversity of arbuscular mycorrhizal fungi of pumpkins Cucurbita spp. under the influence of fertilizers in ferralitic soils of Cameroon and Benin. Journal of Applied Biology and Biotechnology, 3(05): $\quad$ 001-010. DOI: 10.7324/JABB.2015.3501.

Oehl F, Sieverding E, Ineichen K., Mäder P, Boller T, Wiemken A. 2003. Impact of land use intensity on the species diversity of arbuscular mycorrhizal fungi in agroecosystems of central Europe. Applied and Environmental Microbiology, 69(5): 2816-2824. DOI: 10.1128/AEM.69.5.2816-2824.2003.

Saïdou A, Etèka AC, Amadji GL, Hougni DGJ, Kossou D. 2012. Dynamique des champignons endomycorhiziens dans les jachères manioc sur sols ferrugineux tropicaux du Centre Bénin. Annales des Sciences Agronomiques, 16(2): 215-228.

Saïdou A, Kossou D, Acakpo C, Richards P, Kuyper TW. 2012. Effects of farmers' practices of fertilizer application and land use types on subsequent maize yield and nutrient uptake in central Benin. International Journal of Biological and Chemical Sciences, 6(1): 365-378. DOI: http://dx.doi.org/10.4314/ijbcs.v6i1.32.

Saïdou A, Kossou D, Azontondé A, Hougni DGJ. 2009. Effet de la nature de la jachère sur la colonisation de la culture subséquente par les champignons 
endomycorhiziens: cas du système 'jachère' manioc sur sols ferrugineux tropicaux du Bénin. International Journal of Biological and Chemical Sciences, 3(3): 587-597.

Sanchez PA, Jana BA. 2001. Soil fertility replenishment takes off in East and southern Africa. In Intagrated Plant Nutrient Management in Sub-saharan Africa: from Concept to Pratice, Vanlauwe B, Diels J, Sanginga N, Merck (eds). CAB International: Wallingford, UK; 23-45. DOI: 10.1079/9780851995762.0023.

Smith SE, Read DJ. 2010. Mycorrhizal Symbiosis (Second edition). Academic Press, Harcourt Brace and Company Publishers; 605p.

Tchabi A, Coyne D, Hountondji F, Lawouin L, Wiemken A, Oehl F. 2008. Arbuscular mycorrhizal fungal communities in sub-Saharan savannas of Benin, West Africa, as affected by agricultural land use intensity and ecological zone. Mycorrhiza, 18: 181195. DOI: $10.1007 / \mathrm{s} 00572-008-0171-8$.

Trouvelot A, Kough JL, Gianinazzi-Pearson V. 1986. Mesure du taux de mycorhization VA d'un système radiculaire. Recherches et méthodes d'estimation ayant une signification fonctionnelle. In Aspects Physiologiques et Génétiques des Mycorhizes, Dijon, 1985. INRA éd.; 217-221.

Trouvelot A, Gianinazzi-Pearson V, Gianinazzi S. 1982. Les endomycorhizes en agriculture ; recherches sur le blé. In Les Mycorhizes: Biologie et Utilisation. INRA Publ. ed. Les colloques de l'INRA, n²13, Dijon, France ; 251-255. 